RESEARCH HIGHLIGHTS

\title{
A gift from afar...
}

The characterization of two proteins that are involved in the cytoskeletal architecture of the bacterium Prosthecobacter dejongeii has provided a pervasive example of potential horizontal gene transfer from a eukaryotic cell to a prokaryote.

The eukaryotic cytoskeleton contains microtubules composed of $\alpha \beta$ tubulin heterodimers, proteins that are thought to be unique to eukaryotes. The bacterial homologue of $\alpha \beta$ tubulin is the protein FtsZ, which is evolutionarily distant to $\alpha \beta$-tubulin, as indicated by the weak sequence and structural similarities. However, genomic analysis of $P$. dejongeii revealed the presence of two genes, $b t u b A$ and $b t u b B$, that shared significantly higher sequence similarity with the eukaryotic $\alpha \beta$-tubulin than with the bacterial homologue FtsZ. Now, writing in a recent issue of Proc. Natl Acad. Sci. USA, Jan Löwe and colleagues describe the structural and biochemical characterization of BtubA and BtubB. Biochemical investigation of the proteins indicated that some of their properties, including protein folding and dimerization, resembled those of FtsZ rather than $\alpha \beta$-tubulin; chaperones are not required for proper folding and BtubA and BtubB do not form a tight heterodimer. However, when the authors solved the crystal structures of BtubA and BtubB, they were shown to be strikingly similar to the structure of eukaryotic tubulin. Indeed, the structure of BtubA/B is much closer to $\alpha \beta$-tubulin than to FtsZ. Furthermore, the BtubA/B structure contains elements such as surface loops and specific domains that are only necessary in a eukaryotic context.

Taken together, this analysis suggests that the most plausible explanation for the origin of $b t u b A$ and $b t u b B$ - the only genes in the $P$. dejongeii genome with a high degree

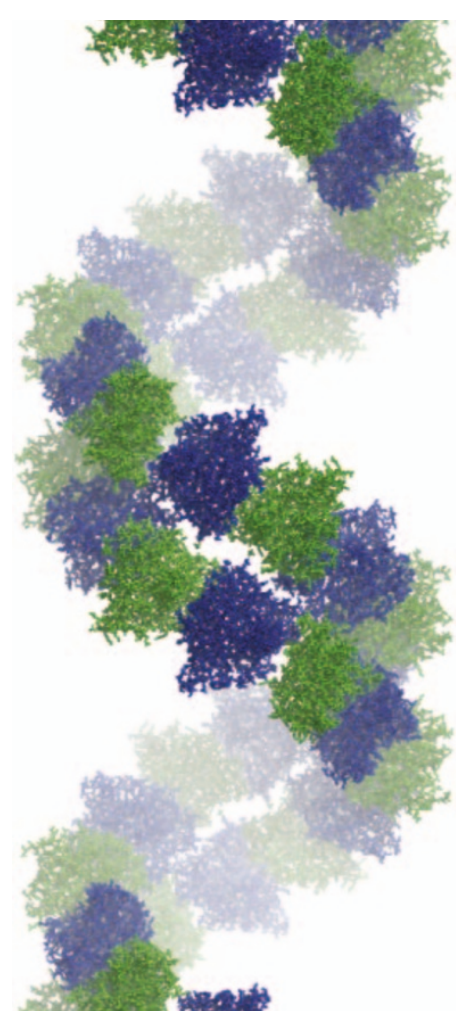

Image courtesy of D. Schlieper, Medical Research Council, Cambridge, UK of similarity to eukaryotic genes - is that tubulin gene(s) were horizontally transferred to Prosthecobacter from a eukaryotic cell.

David O'Connell

(4) References and links ORIGINAL RESEARCH PAPER Schlieper, D. et al. Structure of bacterial tubulin BtubA/B: evidence for horizontal gene transfer. Proc. Natt Acad. Sci. USA 102, 9170-9175 (2005) 